\title{
MULTIDETECTOR COMPUTED TOMOGRAPHY OF RENAL VASCULATURE. ANATOMY AND NORMAL VARIANTS.
}

\author{
Ahmad Hassan MD, AFSA, DIU ${ }^{1}$, Tarek ElKammash MD ${ }^{1}$ and Adel Alam MD'. \\ ${ }^{1}$ Radiology department. Associate Professor of Radiodiagnosis, Suez Canal University. \\ ${ }^{2}$ Department of Urology, Farwania Hospital, Kuwait. Professor of Urology, Banha University.
}

\begin{abstract}
Multi Detector Computed Tomography Angiography (MDCTA) provides a fast, non-invasive modality for the evaluation of the renal vascular pedicle. CTA can reliably and accurately depict the renal arteries and veins and approaches conventional angiography in the assessment of most vascular abnormalities. Knowledge of the variations in renal vascular anatomy is important before laparoscopic donor or partial nephrectomy and vascular reconstruction for renal artery stenosis or abdominal aortic aneurysm.

Objective: To illustrate the diagnostic accuracy of MDCT for evaluation of renal vasculature anatomy.

Design: An analytic, comparative study.

Subjects: fifty six patients including 43 living renal donors, 13 hypertension and abdominal pain (27 men and 29 women, mean age 39.5 years) were examined from January 2008 to September 2010 in Radiology department, Farwania hospital Kuwait. Gold standard was the operative data, conventional angiography or DSA.

Main outcome: The value of MDCT in assessment of renal vascular anomalies.

Results: Renal vascular anatomical variants included multiple arteries $(21.4 \%)$, multiple veins $(7.1 \%)$, early arterial bifurcation $(8.9 \%)$, late venous confluence $(7.1 \%)$, circum-aortic renal veins $(7.1 \%)$ and retro-aortic vein $(3.6 \%)$. The sensitivity and specificity of multiple arterial anomalies were $91.7 \%$ and $97.7 \%$, respectively. The sensitivity and specificity of multiple venous anomalies were $85.7 \%$ and $97.6 \%$, respectively.

Conclusion: MDCT is valuable for detection of renal vascular anomalies.

Keywords: Renal artery. Renal vein. Kidney. Multi-detector computed tomography
\end{abstract}

\section{INTRODUCTION}

K nowledge of the variations in renal vascular anatomy is crucial before laparoscopic donor or partial nephrectomy, vascular treatment for renal artery stenosis and open surgical or endovascular treatment for abdominal aortic aneurysm [1]. Traditionally, conventional catheter angiography used to be performed to assess renal vascular anatomy. However, it is an invasive procedure and has limited value in detailed assessment of renal venous anomalies which is important for laparoscopic nephrectomy [2]. Recently, MDCT angiography has become a key imaging investigation for the assessment of the renal vasculature and has challenged the role of conventional angiography ${ }^{[3,4]}$.

MDCT is now routinely used as a non-invasive alternative to catheter angiography for the evaluation of the vascular system ${ }^{[5] .}$ MDCT systems offer shorter image acquisition time, narrower collimation, improved temporal and spatial resolutions, and near isotropic data acquisition, which is advantageous for two- and threedimensional imaging, compared with original single slice spiral CT ${ }^{[5]}$. However, the main drawbacks of MDCTA are the exposure to ionizing radiation and the use of potentially nephrotoxic iodinated contrast material. As such, its use is limited in children and pregnant women and in patients with impaired renal function.

Magnetic resonance angiography is an alternative non-invasive imaging technique which avoids ionizing radiation ${ }^{[6] .}$ However, its spatial resolution is inferior to that of MDCT; it also has less common availability and higher cost. Furthermore, there are some recent concerns about the safety of some gadolinium based contrast agents. Laparoscopic living donor nephrectomy is a less invasive procedure than open donor nephrectomy and offers numerous advantages over conventional open surgery. The advantages of the laparoscopic approach compared with open nephrectomy are reduction in post-operative pain, a shorter recovery time, reduced length and cost of the hospital stay, and a high degree of patient satisfaction. Several technical challenges are associated with the laparoscopic removal of a kidney. Operative visibility and surgical exposure are limited, so preoperative evaluation of the donor's anatomy is critical ${ }^{[7] .}$

\section{AIM OF THE WORK}

The aim of this study was to determine the accuracy of MDCT in evaluation of renal vascular anatomy. Secondary objectives: The accuracy of maximum intensity projection (MIP) images created at a 64section MDCT console with three-dimensional 
(3D)-workstation-generated images for the definition of renal vascular anatomy.

Normal renal arterial anatomy and variants.

Renal arteries typically arise from the aorta at the level of the superior margin of the second lumbar vertebral body (Fig. 1), slightly inferior the origin of the superior mesenteric artery. The right renal artery orifice is usually more superior and antero-lateral than the left. Typically, the right renal artery has a long downward course to the relatively inferior right kidney, whereas the left renal artery has a more horizontal course to the superiorly located left kidney. The main renal arteries divide into anterior and posterior divisions (Fig. 2) that lie anterior and posterior to the renal pelvis. The anterior division branches into four segmental arteries including apical, upper, middle, and lower anterior. The apical and lower anterior segmental arteries supply the anterior and posterior surfaces of the upper and lower renal poles, and the upper and middle segmental arteries supply the remainder of the anterior surface. The posterior division supplies a large portion of the blood flow to the posterior portion of the kidney. The segmental arteries course through the renal sinus and further subdivide into interlobar arteries [8]. At the level of the renal pyramids, the inter lobar arteries divide into arcuate arteries, which parallel the renal contour along the cortico-medullary junction. The arcuate arteries give rise to multiple interlobular arteries. Also, the renal arteries give off inferior adrenal branches, capsular branches, and branches into renal pelvis and proximal ureter ${ }^{[9]}$. About $70 \%$ of the population may be expected to have a single renal artery that originates from the abdominal aorta on each side. Accessory renal arteries are the most common renal vascular variant and are seen in about one-third of the population ${ }^{[9]}$.

In a study of 400 cadaver renal donors with 800 kidneys, Pollak et al. ${ }^{[10]}$ detected that $23 \%$ had double renal arteries, $4 \%$ triple renal arteries, and $1 \%$ quadruple renal arteries. Bilateral multiple renal arteries occur in $10-15 \%$ of the population ${ }^{[10,11]}$. Accessory renal arteries are considered to be persistent embryonic lateral splanchnic arteries ${ }^{[12] .}$ The origins of the accessory renal arteries may be a high or low position of the abdominal aorta. When it originates from a low position, its origin may be near the aortic bifurcation or from the iliac arteries [9]. Most commonly, the accessory arteries originate from the abdominal aorta and supply the inferior pole of the kidney. Rarely, they can arise from the celiac, mesenteric, lumbar, middle colic or middle sacral artery [12]. Accessory renal arteries are categorized according to their course as either polar (piercing the upper or lower pole of the kidney directly) or hilar (entering the kidney at the hilum) [9]. The polar accessory renal arteries are usually smaller but hilar accessory renal arteries are not always smaller than the principal renal arteries. Prehilar (early) branching of the renal artery is a normal variant in which any branch diverges within $1.5-2.0 \mathrm{~cm}$ from the lateral wall the aorta in the left kidney or in retro-caval segment in the right kidney. This variant is important in renal transplantation, because most surgeons usually require at least a 1.5to $2.0-\mathrm{cm}$ length of renal artery before first branching for successful anastomosis. Surgically, the importance and definition of early arterial bifurcation related to the ability to transect the main renal artery and still provide a common arterial cuff proximal to the bifurcation. Early arterial bifurcation was defined as $<1.5 \mathrm{~cm}$ from the origin of the renal artery. The rationale for using this cutoff was to predict the ability to obtain a common arterial cuff when utilizing a 12-mm stapling device to divide and fixate the renal arterial stump ${ }^{[8]}$.

Normal renal venous anatomy and variants:

The left renal vein usually receives the left adrenal, gonadal and lumbar veins and then passes between anterior to the aorta and posterior to the superior mesenteric artery, to enter the medial side of inferior vena cava (Fig. 3). The right renal vein, which is shorter than the left, enters the lateral side of the inferior vena cava typically at the level of first lumbar vertebra and usually receives no tributaries ${ }^{[13] .}$ The average lengths of the renal veins are approximately $6.8-7.5 \mathrm{~cm}$ on the left and 2.5 $2.6 \mathrm{~cm}$ on the right ${ }^{[12]}$. The renal veins usually lie anterior to the renal artery at the renal hilum. Variation of renal venous anatomy is less common than the arteries. Multiple renal veins are the most common venous variant, seen in about $15-30 \%$ of the population, and more common on the right side ${ }^{[14]}$. The late venous confluence is a another venous variant which is diagnosed on the left side when venous branches join within $1.5 \mathrm{~cm}$ from the left lateral wall of abdominal aorta and on the right side when venous branches join within $1.5 \mathrm{~cm}$ of the confluence with the inferior vena cava. The most common anomaly of the left renal vein is the circum-aortic left renal vein, seen in approximately $2-17 \%$ of the population ${ }^{[15] .}$ In circum-aortic renal vein, the left renal vein divides into ventral and 
dorsal limbs that encircle the abdominal aorta. In the presence of a circum-aortic left renal vein, the adrenal vein enters the pre-aortic limb and the gonadal vein enters the retro-aortic limb. The retroaortic left renal vein, seen in $2-3 \%$ of the population, courses posterior to the aorta and empties into the lower lumbar portion of the inferior vena cava ${ }^{[14] .}$ The left adrenal vein and gonadal vein enter into the left renal vein in almost all cases. However, on the right side, the gonadal vein and adrenal vein enter the right renal vein in only $7 \%$ and $31 \%$ of cases, respectively ${ }^{[14]}$. The left adrenal vein drains into the superior aspect of the left renal vein, while the left gonadal vein drains into it inferiorly, lateral to the left adrenal vein. Two left gonadal veins may be seen in about $15 \%$ of cases [14]. A prominent gonadal vein is diagnosed on the left side when the diameter of this vein is $5 \mathrm{~mm}$ or larger ${ }^{[16] .}$ In about59-88\% of the population, the retroperitoneal veins, including the lumbar, ascending lumbar and hemiazygos veins, drain into the left renal vein ${ }^{[17]}$.

\section{PATIENTS \& METHODS}

The subjects in this study comprised 56 patients They underwent 64 MSCT triphasic imaging. The patients included 27 men and 29 women who ranged in age from 21 to 58 years (mean age, 39.5 years).

Ethical considerations: All the patients had given their informed consent to be included in the study, which was conducted in accordance with the principles of the Declaration of Helsinki ${ }^{[18]}$.

Sample type: Comprehensive sample.

Study design: analytic, comparative study.

The intra-operative, conventional angiography and DSA findings from each patient with respect to the renal vasculature constituted the standard of reference for the imaging findings.

\section{Methods:}

CT exams were performed using GE, light speed VCT 64 slices. $100-140 \mathrm{~mL}$ of a nonionic iodinated contrast agent (Omnipaque $350 \mathrm{mg} \mathrm{I} / \mathrm{ml}$, Iohexol, GE, Ireland) at a rate of $3-4 \mathrm{~mL} / \mathrm{s}$ with a bolustriggered technique ${ }^{[4] .}$

\section{MDCT technique}

MDCTA was performed by using a 64-row MDCT system with the protocol acquiring CT data in the arterial, venous and delayed phases. Firstly, an initial scout and plain scan image were obtained.

After fasting for at least 2-3 hour, each patient ingested $750 \mathrm{ml}$. of water during a 15 - to 20-min period before scanning began. An explanation of the
CT angiography procedure and breathing instructions were then offered to each patient. Unenhanced CT of the abdomen was first performed using a 10-mm slice thickness. A bolus-tracking technique with automated scan-triggering software (SmartPrep, GE Healthcare) was used for initiating arterial phase scanning in all of the patients. A region of interest (ROI) was placed in the abdominal aorta at the level of the celiac artery, and a threshold of $125 \mathrm{HU}$ for peak enhancement in the abdominal aorta was arbitrarily selected for triggering arterial phase imaging. The technical parameters of SmartPrep were identical in all groups. This automatic bolus tracking meant that the arterial phase acquisition began 18-27 s after the start of the injection. Venous phase start 55 seconds after injection.

The estimated contrast dose was determined on the basis of patient weight as follows: weight of less than $45 \mathrm{~kg}, 100 \mathrm{ml} ; 45-90 \mathrm{~kg}, 120 \mathrm{ml}$; and greater than $90 \mathrm{~kg}, 140 \mathrm{ml}^{[19] \text {. }}$

A $\mathrm{kVp}$ of 140 was used in all patients to obtain the initial monitoring scans of SmartPrep before triggering arterial phase imaging. Omnipaque was then administered at 3-4 $\mathrm{ml} / \mathrm{s}$ via an 18-gauge cannula placed in an antecubital vein of the arm. During the arterial phase, the abdomen was scanned from the level of diaphragm to the iliac crest while the patient held his or her breath in end expiration. The main acquisition parameters for the arterial phase were: the detector collimation of $16 \times 0.625$ $\mathrm{mm}$, tube voltage of $120 \mathrm{kv}$, tube current of 200 $240 \mathrm{mAs}$, gantry speed of $0.5 \mathrm{~s} /$ rotation ${ }^{[20] .}$ All images were reconstructed with a stand art soft tissue algorithm and transferred to a separate workstation for post-processing. For threedimensional image reconstruction, the volumetric MDCT data sets were processed on a separate workstation (Advanced Workstation 4.2, GE Health care, Milwaukee, Wis.) with multi-planar reformatting.

Renal arterial and venous anatomy were assessed primarily on arterial phase.The reformations included maximum-intensity-projection (MIP), subvolume MIP, and volume-rendered images for depicting the renal arteries ${ }^{[5]}$. A delayed topogram was routinely obtained 5-10 min after IV contrast material administration to define the collecting system and ureters.

\section{Image acquisition:}

The diagnostic accuracy of renal MDCTA depends on the quality of initial raw data acquired during the 
study. Adequate patient preparation, positioning, as well as the proper contrast material injection, are of paramount importance. The optimal anatomic coverage for the arterial phase scan, that is the principal part of the renal MDCT angiography, should include the region between the celiac artery and terminal part of the common iliac arteries. However, in patients with ectopic or transplanted kidney, the coverage can be modified; for this purpose careful pre-procedural evaluation of the patient including medical records should be done.

Slices with a thickness of $1-1.5 \mathrm{~mm}$ are obtained after rapid injection of contrast. For the evaluation of renal venous structures and abdominal viscera whole abdomen is scanned with a section thickness of $5 \mathrm{~mm}$. Ultimately, in renal transplant donors, 5 10 min delayed scans are obtained for the evaluation of the ureters.

Axial source images remain the basis for diagnosis; however, post-processed 2D and 3D reformations contribute significantly for accurate evaluation. The used post-processing techniques are multiplanar and curved planar reformations (MPR and CPR), maximum intensity projection (MIP), and volume rendering (VR). MIP images provide angiography like images with an overview of vascular anatomy and their variable projection angles be used for the interpretation of arterial anatomy and stenotic lesions. MPR and CPR images are particularly useful for correct evaluation of the arterial luminal diameter ${ }^{[21] .}$ VR images can be used for the overall display of the abdominal vasculature and can provide an insight for the interpreter and referring physicians ${ }^{[22,10] .}$ Finally, the axial source images should always be reviewed for possible presence of an accompanying non-vascular pathology.

\section{Image Analysis:}

Two experienced radiologists $(>10$ years experience) review the images. Any difference in diagnostic confidence in the findings on renal anatomy from the 3D workstation images and simple console MIPs was also noted. The observers were blinded to patient demographics and the technical parameters of the examination. The axial images, 2D data set, and 3D data set were evaluated independently using qualitative and quantitative methods. Qualitative evaluation-the following image parameters were assessed: The quality of the axial images was recorded using a 5-point scale $(1=$ unacceptable, 2 = suboptimal, 3 = diagnostically acceptable, $4=$ good, and $5=$ excellent quality), and the visibility of the branch order (first order, second order, or third order) of the superior mesenteric artery (SMA) and renal arteries was recorded. The quality of the 3D reformations was also assessed on a 5 -point scale ( 1 = poor quality, $3=$ diagnostically sufficient quality, $5=$ excellent quality). In addition, the axial images were evaluated for the number of renal arteries supplying each kidney and the branching pattern of the renal arteries. If an accessory renal artery was detected, its diameter and location (superior pole, hilar, or inferior pole) were recorded ${ }^{[21] .}$ Images in real time at frame rates of 10-30 frames per second. The reviewers used source images as well as 3D display images. For 3D CTA, VR techniques were usually used, but MIP was also used as an adjunct display, especially for small vessels. Alternative visualization techniques included reformatted imaging and stereoscopic display for complex vascular anatomy. These techniques were primarily relied on by each reviewer, and the 3D display parameters, including width, level, opacity, and brightness, were chosen subjectively by the individual reviewer. Renal arterial and venous anatomy were evaluated primarily on arterial phase images, but if the renal veins were not enhanced on the arterial phase images, venous phase images were used.

The reviewers recorded the number of renal arteries found on each side. Any branch within $1.5 \mathrm{~cm}$ from the aorta was classified as early branching. For each artery, other associated findings, including the presence of stenosis and calcifications, were recorded. Renal vein anatomy was evaluated for the number of the renal veins and the presence of accessory veins, retro-aortic veins, and circumaortic veins.

The reviewers completed a worksheet independently for each CT examination. The worksheet detailed the numbers of renal arteries, and the presence of early branching was recorded. Renal vein anatomy was also evaluated for the number of renal veins and the presence of accessory veins, retro-aortic veins, and circum-aortic veins. When discrepancies were found between CT and surgical findings, the CT examination was retrospectively analyzed.

\section{Statistical Analysis}

The sensitivity for each observer and technique was calculated, and the statistical analysis for differences of the sensitivities was performed with the McNemar test. Kappa statistics were used to assess inter-observer agreement in the detection of vascular anatomy. SAS statistical software (version 
9.1, 2002-2003; SAS Institute, Cary, NC) was used to calculate the weighted $\kappa$ values and the confidence intervals for sensitivity for their findings. Surgical correlation for the location of primary and accessory renal arteries, early branching of the renal arteries, and renal vein anomaly was made. Sensitivity, specificity, and accuracy for the presence or absence of supernumerary renal arteries, presence or absence of early branching of the renal artery, and presence or absence of renal vein anomalies of 56 donor and patients were calculated on the basis of each reviewer's evaluation. Ninety-five percent confidence intervals for each sensitivity and specificity were obtained. The average sensitivity and specificity of the two reviewers were also calculated. By using the surgical and angiographic findings as the standard of reference, the sensitivity for detection of the renal arteries and veins and variants were calculated for MIPs (from both console and 3D workstation image sets) based on the findings of the two radiologists. The McNemar test for paired data was used to test for differences between the methods in the number of arteries and veins. A $\mathrm{P}$ value of 0.05 or less was considered to indicate a statistically significant difference.

\section{RESULTS}

After thorough clinical examination \& history taking, the patient underwent the MDCTA. In all the patients, MDCT provided the optimum arterial and venous images without any significant motion or artifacts. Gold standard records revealed a total of 12 multiple arteries and after review of console MIPs, radiologists, detected 12 cases, but they failed to detect an accessory renal artery $1.5 \mathrm{~mm}$ in diameter on the left side, which was found during surgery. There was one case where MDCTA suggested 1-2 $\mathrm{mm}$ accessory upper pole arteries, and no upper pole arteries were identified during the procedure. A retrospective review of the CT imaging appeared to show the artery provide perfusion to the kidney. It is unclear whether this vessel represents a small phrenic arterial branch or whether it was small enough to be incidentally divided during the procedure without identification. Renal vascular anatomical variants (Table 1) included multiple arteries (21.4\%) (Fig. 4), multiple veins $(7.1 \%)$ (Fig. 5), early arterial bifurcation $(8.9 \%)$ (Fig. 6), late venous confluence $(7.1 \%)$, circum-aortic renal veins (7.1\%) (Fig. 7) and retroaortic vein (Fig. 8)(3.6\%). The sensitivity and specificity (Table 2 ) of multiple arterial anomalies were $91.7 \%$ and $97.7 \%$, respectively. The sensitivity and specificity of multiple venous anomalies were $85.7 \%$ and $97.6 \%$, respectively.

The mean sensitivity of CT was $(92.9 \%)$ (Table 3) in detection of overall vascular anomalies. However, positive predictive value was $94 \%$ (Table 4). False negative results were the problem of CT in venous anomalies $(5.4 \%)$. About arterial anomalies, MDCT was relatively high specific for detection of accessory arteries (97.7\%) and for arterial bifurcation (100\%).

Two cases of renal artery stenosis were detected (RAS) (3.6\%), two renal malignancies (Renal cell carcinoma proved histologically) (3.6\%), one had an ectopic kidney (1.8\%).

The sensitivity and accuracy for mapping renal vascular anatomy by two readers were $95.8 \%$, interobserver agreement was excellent $(\kappa=0.89-1.00)$. The $95 \%$ confidence interval for sensitivity was also calculated.

Simple MIPs compared well with 3D-workstation images: MIPs from a pre-designed protocol on the scanner console were generated more quickly than similar images from 3D workstations; postprocessing demands are quickly fulfilled at the scanner console itself. The average time to generate simple MIPs at the console was 2 minutes (range, 1-3 minutes), and 11 minutes (range, $7-15$ minutes) to create images at the $3 \mathrm{D}$ workstation. The test for the difference between the detection of the number of arteries and veins, and the anatomy of the collecting system on 64 MDCT console MIPs and 3D workstation images was not statistically significant $(\mathrm{P}>0.74)$.

Secondary to difficulties in differentiating a late venous confluence in close proximity to the inferior vena cava from two adjacent veins draining separately into the IVC, the positive and negative predictive values were $80 \%$ and $98 \%$, respectively. Twelve supernumerary renal arteries (21.4\%; two arteries and no three arteries were found) to 56 donor kidneys and patients are found. The accuracy was $96.4 \%$. One artery was not identified. None of the CT errors seriously affected the surgical procedure.

A small accessory renal artery to a donor kidney was missed by the two reviewers. This patient, had two renal arteries to the left kidney with a diminutive left upper pole artery found at surgery. The artery was not seen even in retrospect but was not thought by the surgeon to be significant. That artery was seen in retrospect as a small artery of less 
than $2 \mathrm{~mm}$ in diameter. It was not thought by the surgeon to be significant. Early branching was shown in 5 renal arteries, all of which were seen in the left renal artery. Sensitivity and accuracy of CT were $100 \%$ and $100 \%$, respectively. The kappa value of the two reviewers for the presence of early branching of the renal arteries was 0.74

Renal vein anomalies were present in 14 kidneys $(24.9 \%)$, all of which were the left kidney. Sensitivity and accuracy of CT were $91.4 \%$ and $97.8 \%$, respectively. The kappa value of the two reviewers for the presence of renal vein anomalies was 0.85 . Among these fourteen kidneys with renal vein anomalies, four had circum-aortic renal veins and two had retro-aortic renal veins.

Qualitative Assessment: In all cases, the image quality of console MIPs was consistently rated as either good (grade 4) or excellent (grade 5) by the radiologists on a five-point scale (mean grade $=$ 4.4). The diagnostic confidence of these assessors was either high (grade 4) or excellent (grade 5) for these cases (mean grade $=4.6$ ). Console MIPs were graded equal to that $3 \mathrm{D}$ workstation images for diagnostic confidence. Adequacy of console MIPs for surgery was rated as good (grade 4) to excellent (grade 5) by radiologists and surgeons on both image sets on all occasions except for two donor console MIPs and their corresponding 3D workstation images, which were rated as acceptable (grade 3) for surgery. These two donors had larger body size and the CT data sets had greater than expected image noise for the selected noise index. The radiologists reviewed transverse images carefully to confirm early arterial branching.

Table (1): Types and frequency of anatomical variants observed on MDCT in comparison with gold standard findings.

\begin{tabular}{lll}
\hline Anatomical variants & Predicted by MDCT, No, (\%) & Gold standard, No, (\%) \\
\hline Multiple arteries & $12(21.4)$ & $12(21.4)$ \\
\hline Early arterial bifurcation & $5(8.9 \%)$ & $5(8.9)$ \\
\hline Late venous confluence & $4(7.1 \%)$ & $3(5.3)$ \\
\hline Multiple veins & $4(7.1)$ & $2(3.6)$ \\
\hline Circum-aortic renal vein & $4(7.1)$ & $4(7.1)$ \\
\hline Retro-aortic renal vein & $2(3.6)$ & $3(5.3)$ \\
\hline Total & $31(55.4)$ & $29(51.8)$ \\
\hline
\end{tabular}

Table (2): The accuracy, sensitivity, specificity, positive and negative predictive values of MDCT in different anatomical variants.

\begin{tabular}{llllll}
\hline Anatomical variants & Accuracy & Sensitivity & Specificity & PPV & NPV \\
\hline Multiple arteries & $96.4 \%$ & $91.7 \%$ & $97.7 \%$ & $91.7 \%$ & $97.7 \%$ \\
\hline Early arterial bifurcation & $100 \%$ & $100 \%$ & $100 \%$ & $100 \%$ & $100 \%$ \\
\hline Late venous confluence & $96.4 \%$ & $80 \%$ & $98 \%$ & $80 \%$ & $98 \%$ \\
\hline Multiple veins & $94.6 \%$ & $85.7 \%$ & $97.6 \%$ & $92.3 \%$ & $95.3 \%$ \\
\hline Circum-aortic renal vein & $100 \%$ & $100 \%$ & $100 \%$ & $100 \%$ & $100 \%$ \\
\hline Retro-aortic renal vein & $100 \%$ & $100 \%$ & $100 \%$ & $100 \%$ & $100 \%$ \\
\hline
\end{tabular}

Table (3): accuracy, sensitivity and specificity of MDCT in all arterial and venous variants.

\begin{tabular}{llll}
\hline Anatomical variants & Accuracy & Sensitivity & Specificity \\
\hline Arterial variants & $98.2 \%$ & $95.9 \%$ & $98.9 \%$ \\
\hline Venous variants & $97.8 \%$ & $91.4 \%$ & $98.9 \%$ \\
& & & \\
\hline
\end{tabular}


Table (4): True and False positive and negative in different anatomical variants.

\begin{tabular}{lllll}
\hline Anatomical variants & True +ve & True -ve & False +ve & False -ve \\
\hline Multiple arteries & $19.6 \%$ & $76.8 \%$ & $1.8 \%$ & $1.8 \%$ \\
\hline Early arterial bifurcation & $8.9 \%$ & $91.1 \%$ & $0 \%$ & $0 \%$ \\
\hline Late venous confluence & $7.1 \%$ & $89.3 \%$ & $1.8 \%$ & $1.8 \%$ \\
\hline Multiple veins & $21.4 \%$ & $73.2 \%$ & $1.8 \%$ & $3.6 \%$ \\
\hline Circum-aortic renal vein & $7.1 \%$ & $92.3 \%$ & $0 \%$ & $0 \%$ \\
\hline Retro-aortic renal vein & $3.5 \%$ & $96.4 \%$ & $0 \%$ & $0 \%$ \\
\hline
\end{tabular}

\section{Illustrated Figures}
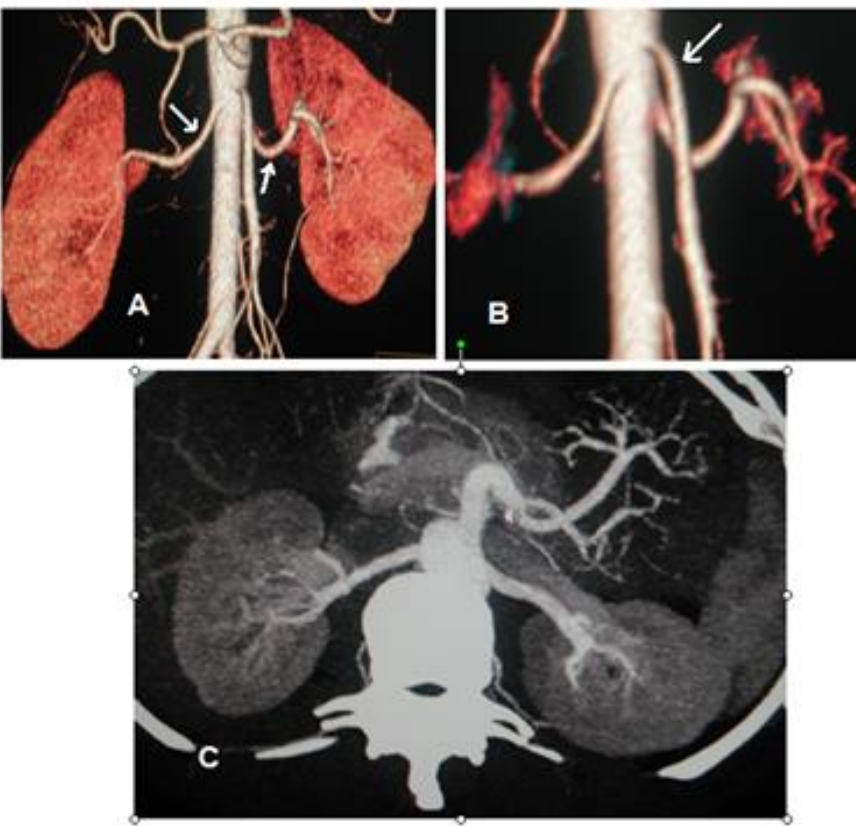

Fig 1
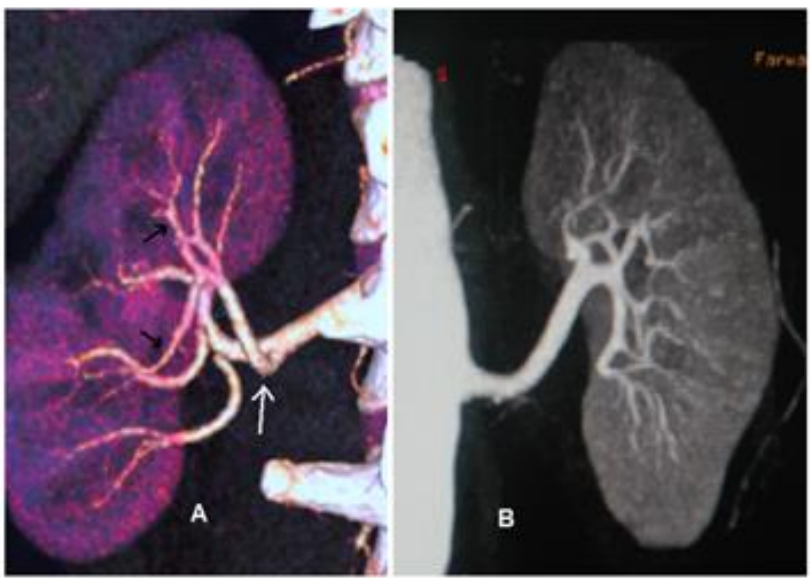

Fig 2
Fig. (1): (A)VR image demonstrates the normal anatomy of the renal arteries (arrows). The renal arteries typically arise from the aorta at the level of L 1-L 2 intervertebral disk space below the origin of the SMA (B)(arrow). (C) Axial MIP

demonstrates renal arteries.
Fig. (2): (A) Coronal VR images show the anterior view of normal segmental anatomy of therenal artery. The first division is the posterior branch (arrow). The posterior branch gives two segmental branches (black arrows), supplying the posterior central portion of the kidney. The main renal artery then continues its course before branching into four anterior branches (arrowhead) at therenal hilum. (B) MIP. Coronal image. 

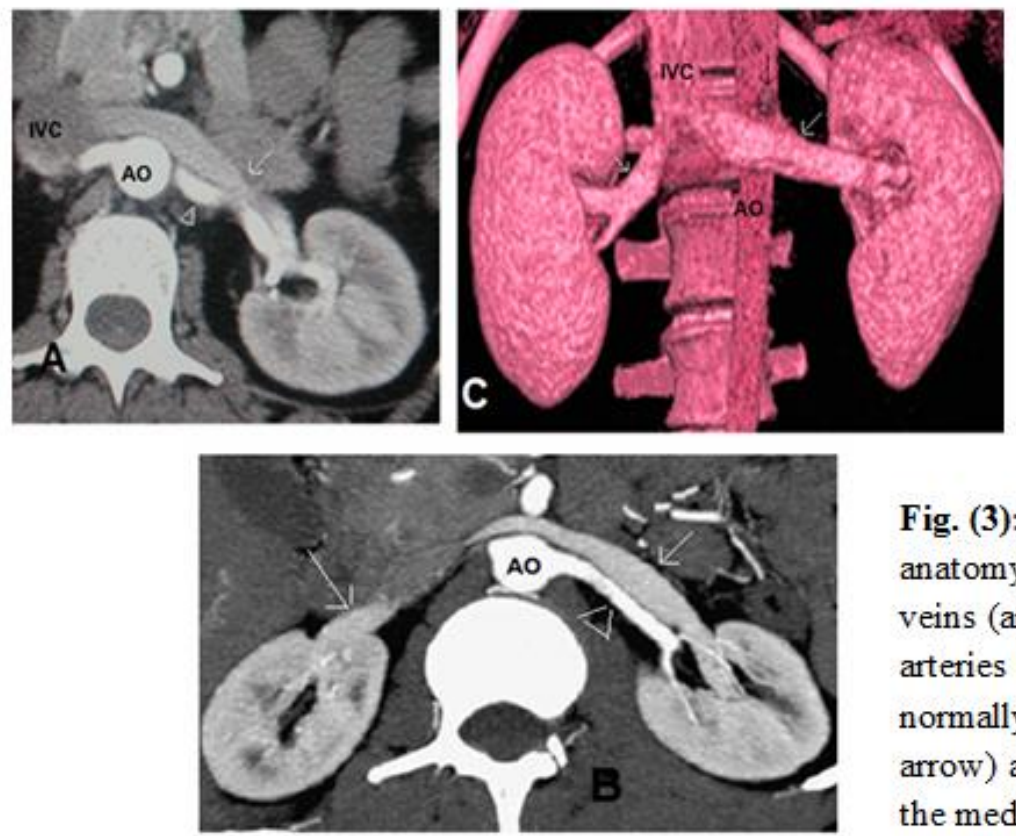

Fig. (3): Images show the normal renal vein anatomy. A, B axial and C, VR: The renal veins (arrows) course anterior to the renal arteries (arrowhead). The left renal vein normally courses between the SMA (black arrow) and the aorta (AO) before draining into the medial aspect of the IVC, while the right vein drains to the lateral aspect. The right renal vein is shorter than the left.

Fig 3
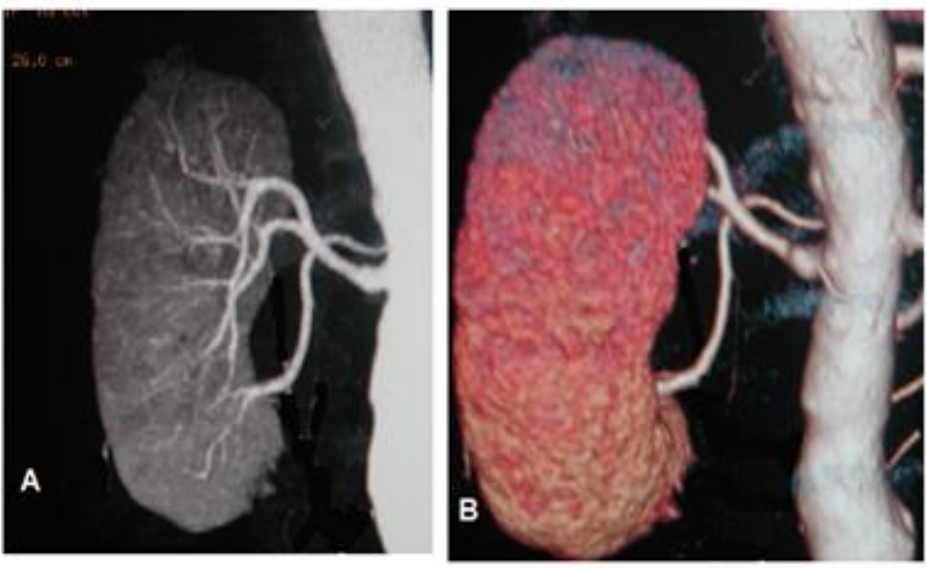

Fig. (4): (A) MIP and (B) VR coronal images show lower polar accessory artery.

Fig 4 


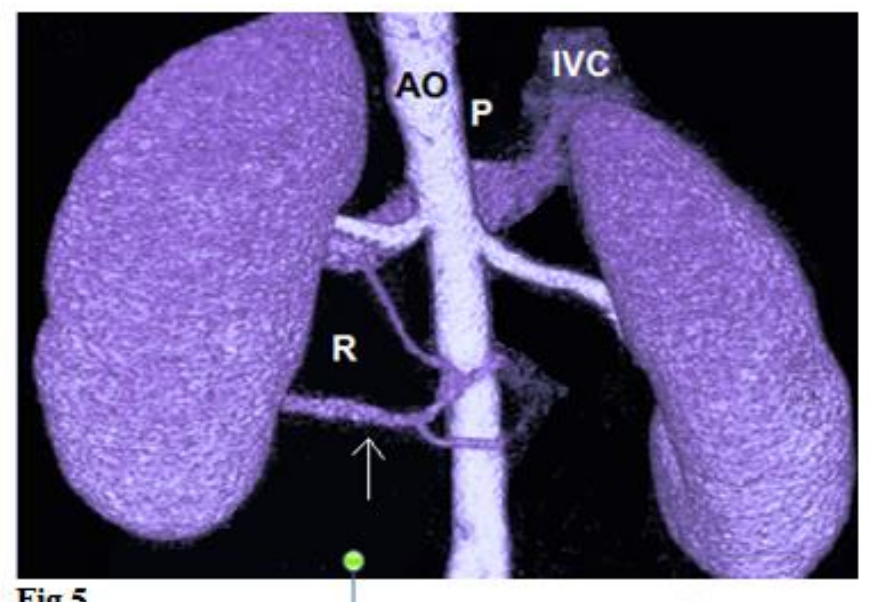

Fig 5

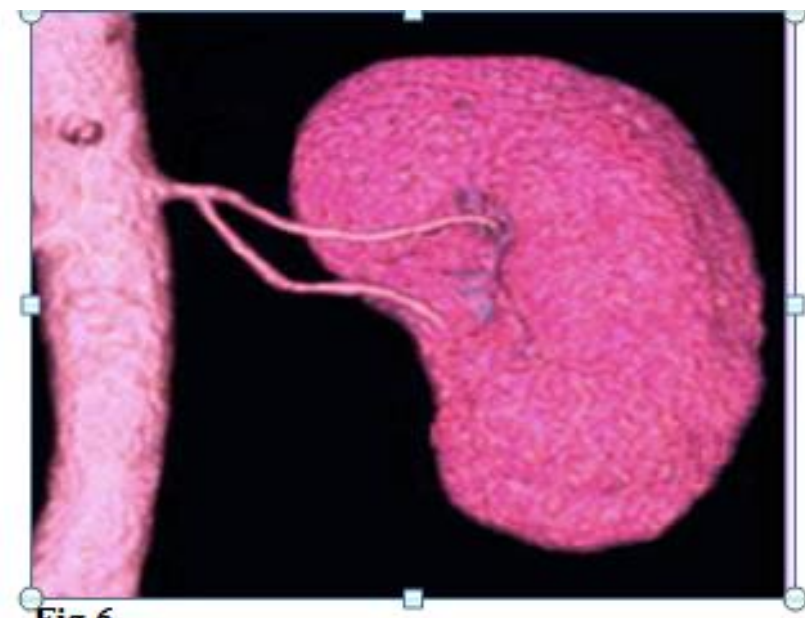

Fig 6

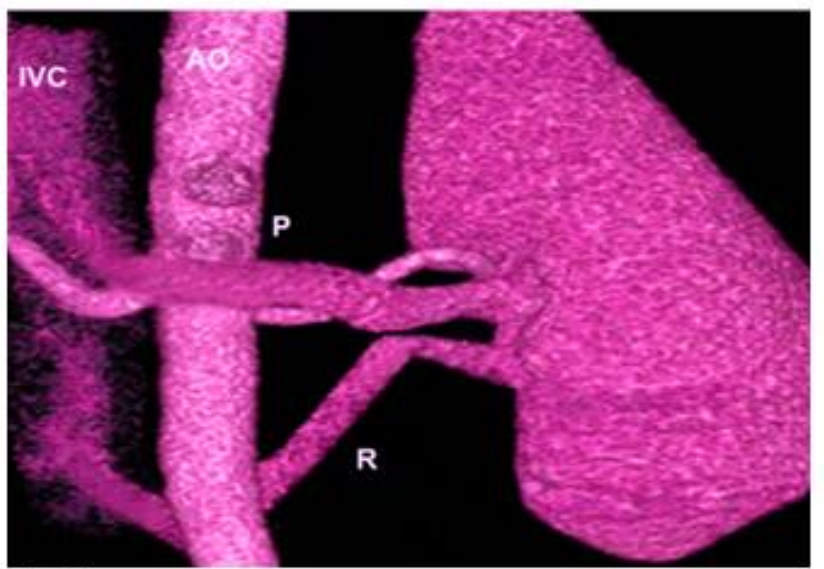

Fig 7
Fig. (5): Posterior coronal VR images show a circum-aortic left renal vein with a pre-aortic component, large one) (P) and a small retro-aortic (R) component.

Fig. (6): coronal VR image shows early branching of the renal artery.

Fig. (7): Coronal volume rendering image shows a circum-aortic left "renal vein with retro-aortic $(\mathrm{R})$ and pre-aortic $(\mathrm{P})$ components. 


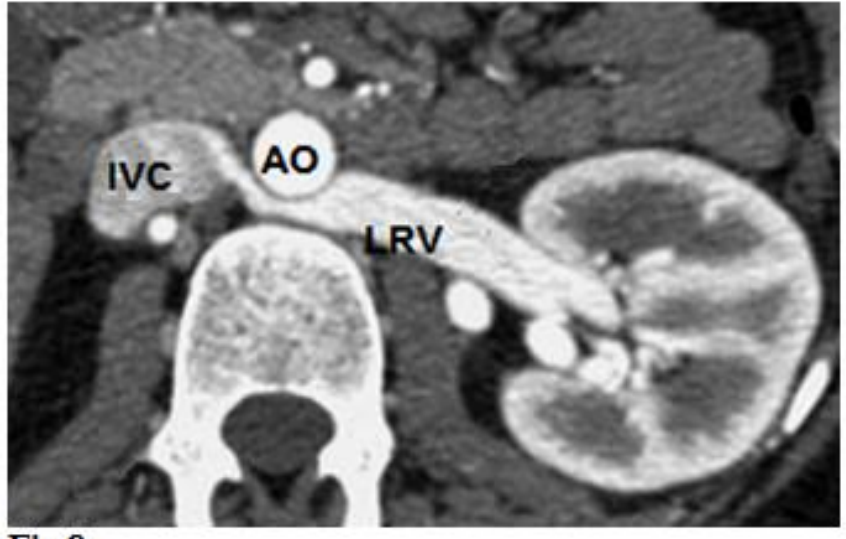

Fig 8

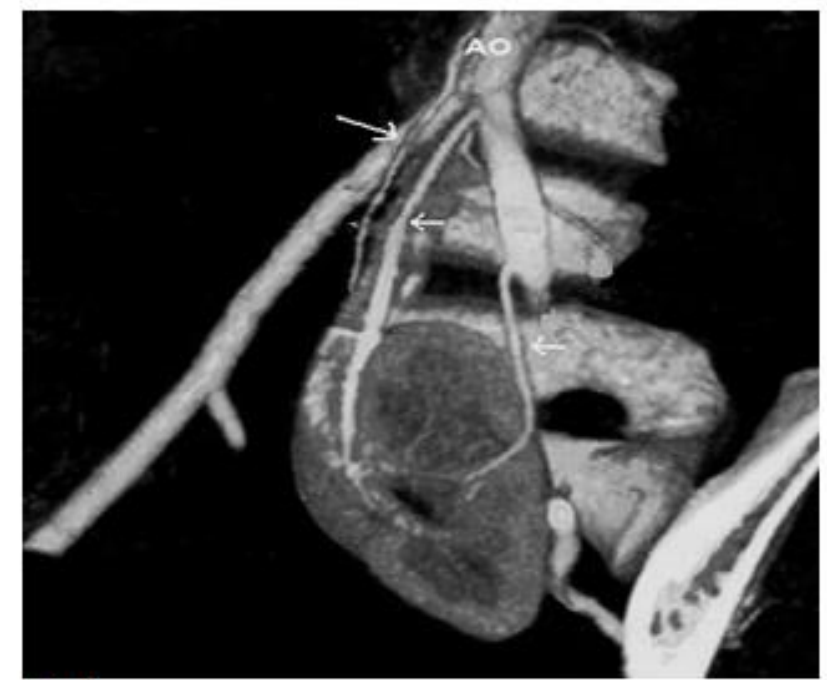

Fig 9

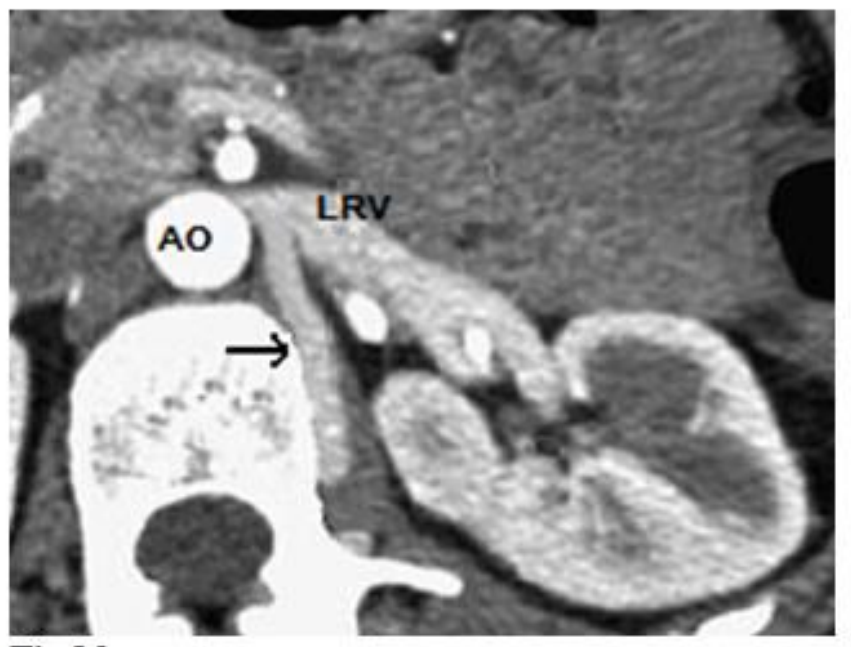

Fig 10
Fig. (8): Axial: shows retro-aortic left renal vein (LRV).
Fig. (9): Volume rendering image shows aberrant renal arteries (arrows) of a pelvic kidney. Two of them originate from the left main iliac artery and the third one from the terminal aorta.

Fig. (10): Axial image shows a prominent left lumbar vein (arrow) entering dorsally into the left renal vein (LRV) 


\section{DISCUSSION}

So far conventional arteriography or DSA has been the gold standard for the evaluation of renal vasculature. But owing to its invasive nature with associated complications, as well as the time and effort involved, catheter angiography is not suited as a screening modality. A wide variety of diagnostic modalities such as duplex and color Doppler sonography, MR angiography, intravenous DSA and of late MDCT angiography are available for this purpose. This study aims to evaluate the role of MDCT in the diagnosis of renal vasculature anatomy. MDCT proved to be an excellent modality for the detection, localization and quantification of renal vascular anatomy. As opposed to angiography, MDCT offers information not only regarding the vessel lumen but also of the vessel wall and the surrounding structures. The ability to view the data set in all three dimensions constitutes an added advantage ${ }^{[6] .}$ A comprehensive anatomic evaluation of potential renal donors is crucial when harvesting is performed using the laparoscopic technique. Multiphase MDCT is an accurate and useful technique for providing a comprehensive preoperative evaluation of renal donors [19, 22,23]. Previous studies showed the incidence of accessory renal arteries, early branching, and venous anomalies to be $25-32 \%, 7-21 \%$, and $7-13 \%$, respectively $[10,11,12]$. In our study, incidence of accessory arteries $(21.4 \%)$, early branching $(8.9 \%)$ and venous anomalies $(25 \%)$. The most common venous anomaly in our study was a circum-aortic left renal vein $(7.1 \%)$. Holden et al. ${ }^{[24]}$ reported 96\% accuracy for MDCT in the evaluation of the renal vascular anatomy for laparoscopic donor nephrectomy. Other investigators have reported the accuracy of MDCT for the detection of renal artery and vein anomalies to be greater than 95\% [25]. In our study, the accuracy of CT is also greater than 95\% (97.9\%).

Laparoscopic nephrectomy is now becoming the surgery of choice for living renal donors. It was introduced in living renal donors in 1995, offering advantages such as less time in hospital, less postoperative pain, few cosmetic concerns and less convalescence time. Thus, preoperative evaluation of these donors using MDCT combines a minimally invasive surgery with minimally invasive imaging giving arterial and venous anatomy with functional capacity of the kidney. Along with arterial anatomy, renal venous anatomy has gained significance; as venous bleeding is a potentially serious complication of laparoscopic surgery ${ }^{[3]}$.

A study by Rubin et al,1995, stated that axial and three-dimensional CT angiography were $100 \%$ sensitive for identifying seven accessory renal arteries and $14 \%$ and $93 \%$ sensitive for identifying five prehilar renal artery branches ${ }^{[26]}$. The capability of MDCT, including fast data acquisition and narrow collimation, is valuable for angiographic applications because of greater anatomic coverage, increased contrast opacification of the arteries, and higher longitudinal spatial resolution. Several reports have shown the accuracy of one-channel CT angiography in establishing the presence of variations or anomalies of renal arteries and veins. The reported accuracy of one-channel CT angiography in detecting accessory arteries, early branching, and renal venous anatomy is in the range of $78-98 \%, 89-99 \%$, and $90-99 \%$, respectively ${ }^{[7]}$. More recently, in a study by Satomi et al, 2003, 18 supernumerary renal arteries to 74 kidneys underwent nephrectomy. MDCT and surgical findings agreed in $93 \%$ of subjects ${ }^{[7] .}$ Sensitivity for the demonstration and location of main renal arteries, however, approaches $100 \%$. Surgical and CT findings correlate in over $95 \%$ of cases ${ }^{[8] .}$

In our study, we missed an accessory artery (1-2 $\mathrm{mm}$ ), this also occurred in other studies, as, in a study of Kulkarni et al, 2011, there were one case where 1-2-mm upper pole vessels were reported, but not seen intra-operatively. It is important to note that in this case, there were adjacent arterial branches supplying the same area of the upper pole, which would in itself an unusual finding. Potential reasons for this discrepancy are that the accessory artery was a phrenic branch that did not supply the kidney or that its small size resulted in inadvertent division which did not correspond to an area of apparent ischemia. Regardless, perfusion was excellent in the upper pole ${ }^{[27] .}$ Other studies stated that limitation for detection occurs with vessels smaller than $2 \mathrm{~mm}$ in size ${ }^{[8]}$. In another study, it mentioned that two small accessory renal arteries were missed by all reviewers. Those arteries were diminutive and were thought to be insignificant by the surgeons ${ }^{[7] .}$ The accuracy of MDCT was $98.2 \%$, however, other studies got higher results, as in this study, whereas CTA had an accuracy of $100 \%$ for diagnosing the number of renal arteries on the side of nephrectomy ${ }^{[5]}$ Zamboni et al, $2010{ }^{[28]}$, reported that MDCT results were accurate in $100 \%$ of arterial and venous anatomical evaluation. Another recent 
study, 2011, that used 64 MDCT, The sensitivity and specificity of multiple arterial anomalies were $100 \%$ and $97 \%$, respectively. The sensitivity and specificity of multiple venous anomalies were $92 \%$ and $98 \%$, respectively [27], in our study, the sensitivity and specificity of multiple arteries were $91.7 \%$ and $97.7 \%$ whereas in multiple venous anomalies, sensitivity was $85.7 \%$ and specificity was $97.6 \%$.

In overall arterial variants, our study presented that sensitivity and specificity were $98.2 \%$ and $98.9 \%$. PPV and NPV were $95.9 \%$ and $98.9 \%$ respectively. This due to $1.8 \%$ of false positive and false negative results. A similar study, Kulkarni et al, 2011, the sensitivity and specificity were $100 \%$ and $97 \%$, while in PPV was $91 \%$ and NPV was $100 \%{ }^{[27] .}$

Early branching of the renal arteries was shown in previous studies as CT and surgical findings agreed in $96 \%{ }^{[7] .}$ The accuracy of early branching in our study was $100 \%$, this is referred to high MDCT resolution capabilities and accurate reconstruction.

The renal venous anatomy is also well demonstrated with CT angiography. The left renal anatomy is especially critical, and this is the preferred side for donation. Tributaries into the left renal vein, especially posterior lumbar branches, are confidently displayed and are of potential surgical importance if noted to be enlarged ${ }^{[8] .}$ Interpretations of late venous confluence involving the right renal vein should be carefully assessed when preoperative surgical planning is conducted. There were four, all on the left side, where there was a discrepancy between the image interpretation and intra-operative finding. In all cases, there was difficulty in identification of a late confluence. One case where a late confluence was not noted and one case in which it was noted, but was not observed intra-operatively. From a surgical perspective, this should be recognized as a potential caveat when evaluating the venous anatomy, pre-operatively. In our studies, accuracy of MDCT in venous anomalies was $97.8 \%$, which is near to other studies results as in Satomi et al; he mentioned that MDCT were $99 \%$ accurate in the evaluation of renal vein anomalies ${ }^{[7]}$. Another relatively old study found that $\mathrm{CT}$ of renal venous anomalies was confirmed in the three patients at surgery $(100 \% \text { accurate })^{[26]}$. This could be due to sampling bias as in more recent studies as in this study 2011, the sensitivity and specificity in detection of venous anomalies were $92 \%$ and $98 \%$ respectively, however, positive predictive value and negative PV were $85 \%$ and
$98 \%$, respectively ${ }^{[27] .}$ In our study, PPV was $93.1 \%$ and NPV was $98.3 \%$.

Volume rendered CT angiography can very quickly and accurately determine the location and course of the renal vascular anatomy. Angioscopic and MIP views provide additional information on the renal vascular anatomy and compliment conventional volume-rendered images. Typically, arterial branches can be confidently identified to at least the segmental level [8]. The MIP technique is excellent for emphasizing high-attenuation structures such as arteries and can provide 3D information if multiple MIPs are generated with different viewing angles and then flipped over or displayed as a cine loop. So, the MIP images are presented in various planes: the straight coronal, oblique coronal parallel to the obliquity of the renal vessels, and thin and betterfocused transverse MIPs with decreased field of view [21]. Our study revealed that MIPs from a predesigned protocol on the scanner console were quicker to generate than similar images from 3D workstations. We believe that 3D workstations are appropriate for complex post-processing demands, like organ volume estimations, while postprocessing demands such as those for renal donors can be quickly fulfilled at the scanner console itself. The post-processing can either take place on the same console after scanning the patient or on a separate 64 multi-detector CT console.

\section{CONCLUSION}

MDCT angiography is an excellent imaging investigation because it is a fast and non invasive tool that provides highly accurate and detailed evaluation of normal renal vascular anatomy and variants. The number, size and course of the renal arteries and veins are easily identified by MDCT angiography.

\section{REFERENCES}

1. Khamanarong K, Prachaney P, Utraravichien A, Tongun T, SripaorayaK: Anatomy of renal arterial supply. Clin. Anat.2004, 17:334-336.

2. Hänninen EL, Denecke T, Stelter L, Pech M, Podrabsky P, Pratschke J et al: Preoperative evaluation of living kidney donors using multi-rowdetector computed tomography: comparison with digital subtraction angiography and intra-operative findings. Transpl. Int. 2005, 18:1134-1141.

3. Rastogi N, Sahani DV, Blake MA, KoDC, Mueller PR: Evaluation of living renal donors: accuracy of three dimensional 16-section CT. Radiology, 2006, 240:137-144.

4. Raman SS, Pojchamarnwiputh S, Muangsomboon K, Schulam PG, Gritsch HA, Lu DSK : Surgically relevant normal and variant renal parenchymal and 
vascular anatomy in preoperative 16-MDCT evaluation of potential laparoscopic renal donors. Am J Roentgenol., 2007,188:105-114.

5. Dushyant V. Sahani, Sanjeeva P. Kalva, Peter F. Hahn and Sanjay Saini: 16-MDCT Angiography in Living Kidney Donors at Various Tube Potentials: Impact on Image Quality and Radiation Dose . AJR 2007; 188:115-120.

6- Monroy-Cuadros M, McLaughlin K, Salazar A, Yilmaz S: Assessment of live kidney donors by magnetic resonance angiography: reliability and impact on outcomes. Clin Transplant, 2008, 22:2931.

7. Satomi Kawamoto, Robert A. Montgomery, Leo P. Lawler, Karen M. Horton and Elliot K. Fishman Multi-detector CT Angiography for Preoperative Evaluation of Living Laparoscopic Kidney Donors. AJR 2003; 180:1633-1638.

8. Aysel Türkvatan, Mustafa Özdemir, Turhan Cumhur, Tülay Ölçer: Multidetector CT angiography of renal vasculature: normal anatomy and variants, Eur Radiol ,2009, 19: 236-244.

9. Kadir S: Angiography of the kidneys. In: Kadir S (ed) Diagnostic angiography. Saunders, Philadelphia, 1986, pp 445-495.

10. Pollak R, Prusak BF, Mozes MF: Anatomic abnormalities of cadaver kidneys produced for purposes of transplantation. Am Surg., 1986, 52:233235.

11. Satyapal KS, Haffejee AA, Singh B,Ramsaroop L, Robbs JV, Kalideen JM: Additional renal arteries: incidence and morphometry. Surg. Radiol.Anat., 2001, 23:33-38.

12. Williams PL, Warwick R, Dyson M, Bannister LH: The urinary organs. In: Williams PL, Warwick R, Dyson M, Bannister LH (eds) Gray's anatomy, 37th ed. Churchill Livingstone, New York,1989, pp 1397 1416.

13. Urban BA, Ratner LE, and Fishman EK: Threedimensional volume rendered CT angiography of the renal arteries and veins: normal anatomy, variants, and clinical applications. Radiographics, 2001, 21:373-386.

14. Abrams HL: Renal venography.In: Abrams HL (ed) Abrams angiography,2nd edn. Little Brown, Boston, 1983, pp 1327-1364.

15. Trigaux JP, Vandroogenbroek S, DeWispelaere JF, Lacrosse M, Jamart J: Congenital anomalies of the inferior vena cava and left renal vein: evaluation with spiral CT. J Vasc. Intervent. Radiol. , 1998, 9:339345 .

16. Kawamoto S, Lawler LP, Fishman EK: Evaluation of the renal venous system on late arterial and venous system on late arterial and venous phase images with MDCT angiography in potential living laparoscopic renal donors. AJR Am. J. Roentgenol., 2005, 184:539-545244.

17. Tuncay Hazırolan, Meryem Öz, Barış Türkbey, Ali Devrim Karaosmanoğlu, Berna Sayan Oğuz, Murat Canyiğit: CT angiography of the renal arteries and veins: normal anatomy and variants. Diagn Interv Radiol 2011; 17:67-73.

18. Declaration of Helsinki: recommendations guiding physicians in biomedical research involving human subjects. Bull Pan Am Health Organ 1990; 24:606 609.

19. Sahani D, Rastogi N, Greenfield A, et al. Multidetector row CT in evaluation of 94 living renal donors by readers with varied experience. Radiology 2005; 235: 905-910.

20. Ertl-Wagner BB, Hoffmann RT, Bruning R: Multidetector row $\mathrm{CT}$ angiography of the brain at various kilovoltage settings. Radiology 2004; 231:528 -535.

21. Anand Kumar Singh, Dushyant V. Sahani, Christopher R. Kagay, Sanjeeva P. Kalva, Mukta C. Joshi, Nahel Elias, et al, Semi-automated MIP Images Created Directly on 16-Section Multi-detector CT Console for Evaluation of Living Renal Donors. Radiology, 2007,244, 583-590.

22. Hänninen E, Denecke T, Stelter L: Preoperative evaluation of living kidney donors using multi-row detector computed tomography: comparison with digital subtraction angiography and intra-operative findings. Transpl Int. 2005; 18:1134-1141.

23. Kawamoto S, Montgomery RA, Lawler LP, Horton KM, Fishman EK. Multi-detector CT angiography for preoperative evaluation of living laparoscopic kidney donors. AJR 2003;180:1633-1638.

24. Holden A, Smith A, Dukes P, Pilmore H, Yasutomi M. Assessment of 100 live potential renal donors for laparoscopic nephrectomy with multi-detector row helical CT. Radiology 2005; 237:973 -980.

25. Giulia A. Zamboni, Janneth Y. Romero and Vassilios D. Raptopoulos: Combined Vascular-Excretory Phase MDCT Angiography in the Preoperative Evaluation of Renal Donors. AJR 2010; 194:145-150.

26. G. D. Rubin, E J Alfrey, M. D. Dake, C. P. Semba, F. G. Sommer, P. C. Kuo :Assessment of living renal donors with spiral CT. 1995 , Radiology, 195, 457462.

27. S. Kulkarni' S. Emre, A. Arvelakis, W. Asch, M. Bia, R. Formica et al: Multi-detector CT angiography in living donor renal transplantation: accuracy and discrepancies in right venous anatomy. Clinical transplantation, Vol. 25, P.77-82, 2011.

28. Zamboni GA, Romero JY and Raptopoulos VD. Combined vascular-excretory phase MDCT angiography in the preoperative evaluation of renal donors. AJR Am J Roentgenol. 2010; 194(1):145-50. 